\title{
Experimental Comparison of Call String and Functional Approaches to Interprocedural Analysis
}

\author{
Florian Martin \\ Universität des Saarlandes \\ P.O. Box 151150, 66041 Saarbrücken \\ martin@cs.uni-sb.de
}

\begin{abstract}
The techniques used to implement (non-trivial) interprocedural data flow analyzers can be generally divided into two subsets: the call string approach and the functional approach as presented in [Sharir and Pnueli, 1981]. Both differ in time and space complexity as well as in precision due to properties of the abstract domains and transfer functions. We have developed a data flow analyzer generator PAG which is able to produce interprocedural analyzers for both techniques. We specified two variants of constant propagation working in an ANSI-C compiler; a copy constant propagation that uses distributive transfer function and can be solved precisely, even interprocedurally [Sagiv et al., 1995], and a full constant propagator which includes an interpreter for expressions of the language. We present the practical relevant results applying both analyzers to a fair set of real-world programs and compare the space/time consumption of the analyzers versus their precision.
\end{abstract}

\section{Introduction}

The need for interprocedural analyses results from a conflict between the goals of software engineering and performance [Metzger and Stroud, 1993]. The usage of procedural and functional abstraction generalizes the code and makes it more extensible and maintainable. Optimizing compilers try to compute information about program points that can be used for code improvements. Intraprocedural analysis assumes the worst case (loss of all information) at procedure boundaries, whereas interprocedural analysis propagates information across procedure/function boundaries or eliminates the calls by inline expansions for nonrecursive calls. There exist two different approaches to interprocedural analysis which have been discussed in [Sharir and Pnueli, 1981]: the call string and the functional approach. The call string approach separates call chains (and the related data flow information) to a procedure differing in a suffix of a fixed length. Thus precision may increase with longer call strings. The maximally useful length for non-recursive programs is the height of the call DAG. For recursive programs the number of possible call strings is infinite. If there are no recursive procedures 
the number of call strings can be exponential in the number of procedures. Thus the call string length has to be limited in any practical implementation to some constant $K$.

The functional approach, on the other side, computes a function for each procedure describing the "abstract" effect of the procedure. These functions are then used in a standard (intraprocedural-like) algorithm. However, the computation of the abstract functions may not terminate for lattices of infinite cardinality, even if they are of bounded height.

We designed and implemented an interprocedural data flow analyzer generator PAG [Martin, 1998], that produces analyzers using either the call string approach with a length parameter or the functional approach. Using this analyzer generator we generated two analyzers, which have been included in a ANSI-C compiler, and measured the efficiency and precision for practical relevant applications.

By creating PAG we hope to close the gap between the theory of abstract interpretation and practical useful analyzers. PAG is available under a free academic license on request from the author or in form of a simplified WWW interface (www.cs.uni-sb.de/ $\sim \operatorname{martin} / \mathrm{pag}$ ).

The paper is organized as follows: a brief introduction to the framework of dataflow analysis is followed by the discussion of the two interprocedural approaches. Then these are compared based on experimental data for two data flow problems.

\section{Techniques}

The data flow analysis practiced nowadays is based on the work described in [Kam and Ullman, 1977]. It is uses a control flow graph $C F G=(V, E)$ that contains a node for every statement or basic block in a procedure and an edge for a possible flow of control. The set of vertices $V$ is a disjoint union of the set of nodes $V_{i}$ for each subroutine $p_{i}$ in the program ${ }^{1}$. Furthermore unique entry nodes $s_{i}$ and exit nodes $e_{i}$ for each procedure $p_{i}$ are added. A data flow analysis problem $(D F P)$ is a combination of such a graph with a complete lattice of values, called the underlying lattice $(\mathcal{L})$, and a family of functions (one for each node). These functions express the local semantics and are called transfer functions $(\llbracket \bullet \rrbracket: \mathcal{L} \rightarrow \mathcal{L})$. If every transfer function is monotone (distributive) the problem is called a monotone (distributive) problem. To describe the solution of a data flow problem the semantics of a path $\pi=n_{1}, \ldots, n_{k}$ in the $C F G$ is defined as:

$$
\llbracket \pi \rrbracket= \begin{cases}i d & \text { if } \pi=\epsilon^{2} \\ \llbracket\left(n_{2}, \cdots, n_{k}\right) \rrbracket \circ \llbracket n_{1} \rrbracket & \text { otherwise }\end{cases}
$$

$\overline{1}$ The existence of a main routine $p_{0}$ is assumed. 
The desired solution of the DFP is the union of the semantics of all paths applied to bottom $(\perp)$, called the merge over all paths solution:

$$
\operatorname{MOP}(n)=\bigsqcup\left\{\llbracket \pi \rrbracket(\perp) \mid \pi \text { is a path from } s_{0} \text { to } n\right\}
$$

for every $n \in V$. As the set of all paths from $s$ to $n$ is usually infinite, this solution is in general not computable. Therefore, the minimal fixed point solution was introduced:

$$
\operatorname{MFP}(n)= \begin{cases}\llbracket n \rrbracket(\perp) & \text { if } n=s_{0} \\ \llbracket n \rrbracket(\bigsqcup\{\mathbf{M F P}(m) \mid m \text { predecessor } n\}) & \text { otherwise }\end{cases}
$$

If $\operatorname{MFP}(n)=\operatorname{MOP}(n)$ for all $n$, then the MFP solution is called precise. Kam has proved in [Kam and Ullman, 1977] that for every monotone data flow problem the MFP is greater (with respect to the ordering of the lattice) than the MOP solution, and is therefore a safe approximation. Moreover, if the DFP is distributive the MFP solution is precise. The interprocedural version of this theorem is presented in [Knoop and Steffen, 1992].

\subsection{Call String Approach}

This approach considers procedure calls and returns as ordinary transfers of control, but avoids propagation along interprocedurally invalid paths. This can be implemented by tagging the propagated data with a call string, which is a sequence of call nodes, whose calls are still unfinished. As the number of call strings may be infinite their length is bounded by a constant $K$. This results in an approximate solution by merging the information belonging to call strings with the same $K$-suffix. For a more practical implementation a vector of data flow elements is used. If a call site $c$ has to be appended to a call string $\gamma=c_{1} c_{2} \ldots c_{K}$ which already has length $K$ the first element $c_{1}$ of $\gamma$ is removed, which results in $c_{2} \ldots c_{K} c$. This method is turned into a vector based implementation by encoding the call strings as numbers which will correspond to positions in the data flow array: assign to every call site $c$ a unique number between one and $M-1$, where $M$ is the number of call sites in the given program plus one. Then a call string $\gamma=c_{i_{1}} \ldots c_{i_{n}}$ corresponds to a $K$ digit number $m$ to base $M\left(m=i_{1} \ldots i_{n}\right)$. Converting this number to the decimal system is done in usual way: multiply the $n$-th digit by $M^{n}$ and add this for all digits $\left(m^{\prime}=\sum_{1 \leq j \leq n} i_{j} * M^{j}\right)$. This results in a number between zero and $M^{K}$ where zero denotes the empty call string $\epsilon$. Fortunately not all of these call strings are valid for each procedure. So all invalid numbers can be omitted for a fixed procedure and the array can be compressed, so that every position is used.

As an example consider the flow graph from the left side of Fig. 1 with $K=1$. Valid call strings for proc are $c_{1}, c_{1} c_{2}, c_{1} c_{2} c_{2}, \ldots$ If they are restricted to length one $c_{1}$ and $c_{2}$ remain. For main the empty call string is the only valid call string. So in the procedure main position zero of the data flow array of length three is

\footnotetext{
${ }^{2} \epsilon$ denotes the empty path.
} 

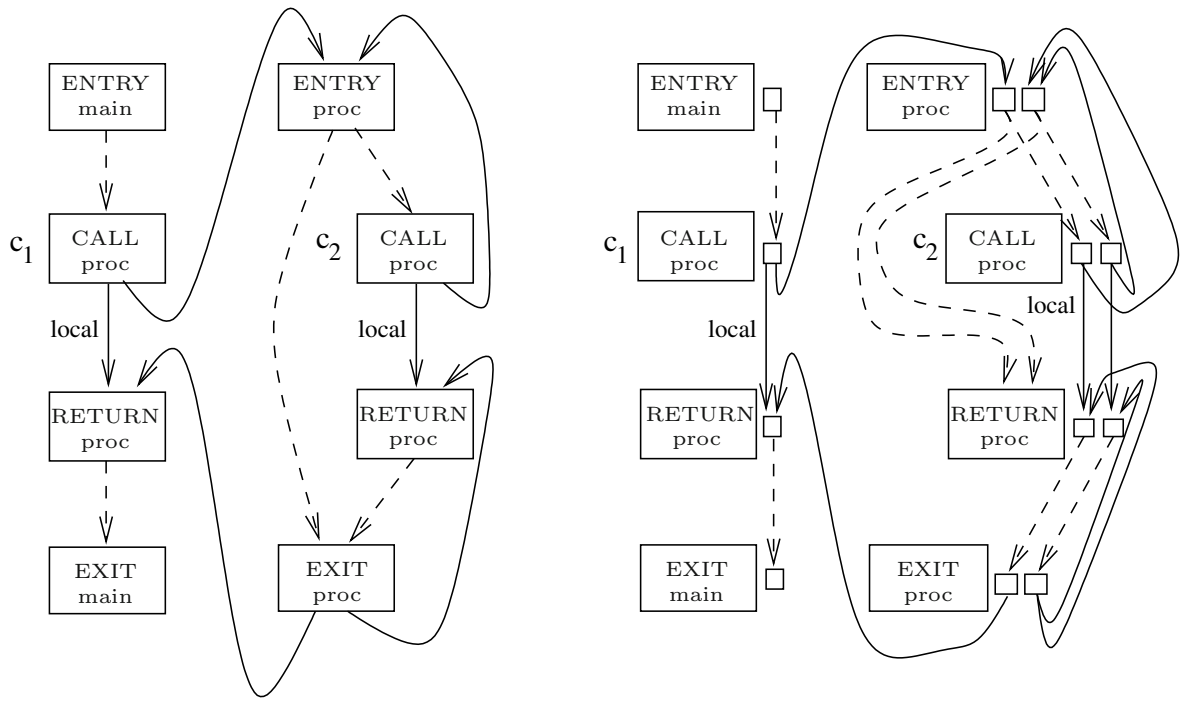

Fig. 1. Example of the vector based call string approach with $K=1$

used and in procedure proc the positions one and two are used. This results in the graph on the right side of Fig. 1.

If the underlying lattice $\mathcal{L}$ is finite a vector implementation can be constructed that results in the precise solution of the DFP. Call chains of length up to $|\mathcal{L}|^{2} * M$ must be separated.

The vector based approach can also be used to implement more sophisticated methods which can also be used for the analysis of loops [Martin et al., 1998].

\section{$2.2 \quad$ Functional Approach}

The functional approach computes for every procedure in the program a function which maps the data flow value at the entry of the procedure to the corresponding data flow value at the exit of the procedure. Because a procedure can be simultaneously recursive it is necessary to do this computation for all procedures in an interleaved fashion. The function is computed by tabulating input/output pairs. This table is guaranteed to be finite if the lattice is of finite cardinality. As an optimization only those input/output pairs are computed for each procedure where the input value actually occurs.

Now a more formal description of this method is given: For each procedure $p_{i}$ and each node $n \in V_{i}$ we define an element $\Phi\left(s_{i}, n\right): \mathcal{L} \rightarrow \mathcal{L}$ which describes the effects to elements of $\mathcal{L}$ when they are propagated from the start $s_{i}$ of procedure $p_{i}$ to $n$. These functions are defined by:

$$
\begin{aligned}
& \Phi\left(s_{i}, s_{i}\right)=\llbracket s_{i} \rrbracket \\
& \Phi\left(s_{i}, n\right)=\sqcup_{(m, n) \in E} \mathcal{E}(n) \circ \Phi\left(s_{i}, m\right)
\end{aligned}
$$




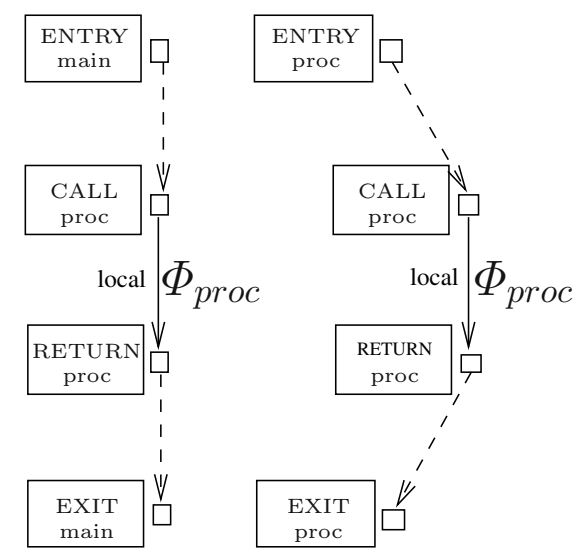

Fig. 2. Functional Approach

and $\mathcal{E}$ is defined as either the normal transfer function for intraprocedural edges or $\Phi$ for interprocedural ones:

$$
\mathcal{E}(n)= \begin{cases}\llbracket n \rrbracket & \text { if } \forall j: n \neq s_{j} \\ \Phi\left(s_{j}, e_{j}\right) & \text { if } \exists j: n=s_{j}\end{cases}
$$

The results of the above equation system are used to compute the solution of the data flow problem:

$$
\begin{aligned}
& \mathcal{F}\left(s_{\text {main }}\right)=\llbracket s_{\text {main }} \rrbracket(\perp) \\
& \mathcal{F}\left(s_{i}\right)=\sqcup_{\left(c, s_{i}\right) \in E} \mathcal{F}(c) \\
& \mathcal{F}(n)=\Phi\left(s_{j}, n\right)\left(\mathcal{F}\left(s_{j}\right)\right) \text { for } n \neq s_{j}, n \in V_{j}
\end{aligned}
$$

The iteration algorithm in PAG is implemented with some optimizations that are specially developed to handle large equation systems with a dynamic number of variables (the elements in the abstract function tables correspond to the variables, so their number is dynamic as the size of the tables is determined in a demand driven way). These optimizations turned out to be very good for this class of equation systems (cf. [Fecht, 1997,Nielson et al., 1998]).

\subsection{Comparison}

We briefly summarize the properties of both approaches (assuming a language with recursive procedures and table based implementation of the functional approach) for a given lattice $\mathcal{L}$. While the call string approach computes only values of $\mathcal{L}$, the functional approach computes values from $\mathcal{L} \rightarrow \mathcal{L}$. In order to guarantee termination it is required that this function space is finite. Thus the call string approach is always guaranteed to terminate, but may deliver worse results. Additionally there is the problem of finding an appropriate length $\mathrm{K}$. 
The functional approach is guaranteed to terminate for finite lattices, but may be exponential in the size of the lattice. It delivers best results.

An other point for the reliable use in a compiler is the the speed of the analysis. The speed for interprocedural analyses depends mainly on how often the procedures of the programs are visited during the analysis. In the $K$ call string approach this is the number of $K$-paths to the procedure in the call graph. In the functional approach it is the number of different data flow values that occur at the beginning of the procedure. In order to keep this number small one tries usually to benefit from the locality properties of the programming language: if it is known that a procedure $p$ can only read and write a certain part of the data flow information, the rest of the data flow information is passed directly from the call to the return of the procedure. This is modeled in PAG by the local edge from Fig. 1 and 2.

But for a constant propagation in $\mathrm{C}$ one can not bypass any part of the data flow information since any procedure can modify any other variable through pointer accesses. This is different for other analyses and programming languages. E.g. in Pascal all variables local to the caller can be passed by any call.

\section{Practical Evaluation}

We generated data flow analyzers for copy constant propagation (ccp) which is a distributive problem and for full constant propagation ( $f p$ ) which is not. $\mathcal{L}_{c c p}$ is finite and therefore of bounded height and the $\mathcal{L}_{f p}$ is infinite but has bounded height. The ccp only processes assignments of the form $x:=y$ or $x:=c$ where $x, y$ are variables and $c$ is a constant, whereas $f p$ includes a full interpreter for expressions. Furthermore, we use a simplified form of a technique called downset completion [Cousot and Cousot, 1992]; instead of combining the data flow information directly where two (or more) control flow edges come together, we first apply the transfer function and combine the results: $f(a \sqcup b)$ is replaced by $f(a) \sqcup f(b)$. This results in better solutions for non distributive $\sqcup$.

Figure 3 shows the structure and size of the used test programs in ANSI C, where most of them are well known everyday life programs $\left(\mathrm{a}^{6 *}\right.$ ) indicates programs with procedural variables). The columns give from left to right: the name of the program, a short description, the number of lines without comments, the number of procedures, the number of control flow nodes, and the number of variables.

For each of the programs the functional analyzer was applied. It finds the precise solution for any program in case of the copy constant propagation. This is also true for the full constant propagation, but the termination of the functional analyzer is not guaranteed. Also the call string analyzers have been applied with call strings up to length two or less if they find the same number of constants as the functional approach.

For each program four mayor columns are printed in Fig. 4 for the copy constant propagation and in Fig. 5 for the full constant propagation: three for call string approach of length zero, one, and two $\left(\mathcal{C}_{0}, \mathcal{C}_{1}, \mathcal{C}_{2}\right)$, and one for the 


\begin{tabular}{|l|l|r|r|r|r|}
\hline program & description & lines & flow nodes & procedures & variables \\
\hline \hline bison & parser generator & 6438 & 11722 & 155 & 3575 \\
\hline cdecl & C++ compiler part & 2831 & 2401 & 33 & 841 \\
\hline cloop & benchmark & 1488 & 2176 & 26 & 622 \\
\hline dhry & dhrystone & 446 & 319 & 14 & 240 \\
\hline ed & editor & 1506 & 2745 & 47 & 796 \\
\hline flex & scanner generator & 5985 & 8688 & 129 & 2314 \\
\hline flops & benchmark & 723 & 353 & 3 & 139 \\
\hline gzip & compress & 4056 & 6043 & 47 & 796 \\
\hline linpack & benchmark & 821 & 796 & 13 & 278 \\
\hline twig & code generator & 2092 & 3198 & 81 & 1085 \\
\hline xmodem & communication & 2060 & 2965 & 31 & 1159 \\
\hline
\end{tabular}

Fig. 3. The set of test programs

\begin{tabular}{|c|c|c|c|c|c|c|c|c|c|c|c|c|}
\hline \multirow[b]{2}{*}{ Prog } & \multicolumn{3}{|c|}{$\mathcal{C}_{0}$} & \multicolumn{3}{|c|}{$\overline{\mathcal{C}_{1}}$} & \multicolumn{3}{|c|}{$\mathcal{C}_{2}$} & \multicolumn{3}{|c|}{$\mathcal{F}$} \\
\hline & Prec & Fold & Time & Prec & Fold & Time & Prec & Fold & Time & Prec & Fold & Time \\
\hline cdecl & 615 & 10 & 15,66 & 627 & 10 & 66,41 & & & & 627 & 10 & 113,93 \\
\hline twig & 756 & 6 & 6,83 & 865 & 6 & 45,13 & 891 & 6 & 308,22 & 993 & 6 & $|1746,14|$ \\
\hline dhry & 81 & 2 & 1,82 & 109 & 2 & 1,54 & 109 & 2 & 1,45 & 109 & 2 & 2,06 \\
\hline ed & 1098 & 42 & 7,81 & 1175 & 44 & 26,04 & 1198 & 44 & 37,05 & 1198 & 44 & 27,25 \\
\hline cloop & 9839 & 145 & 20,95 & 9865 & 145 & 200,30 & & & & 19649 & 145 & 6309,13 \\
\hline flex & 3362 & 22 & 16,40 & 3496 & 22 & 88,76 & $\mid 9714$ & 22 & 378,66 & 9731 & 22 & 193,54 \\
\hline flops & 6897 & 107 & 0,52 & 7973 & 119 & 0,50 & & & & 7973 & 119 & 0,70 \\
\hline bison & 2699 & 10 & 24,45 & 2974 & 10 & 157,12 & $\mid 2978$ & 10 & 372,43 & 2978 & 10 & 303,74 \\
\hline linpack & 355 & 78 & 5,17 & 355 & 78 & 17,60 & 355 & 78 & 24,36 & 355 & 78 & 5,72 \\
\hline gzip & 2744 & 16 & 9,52 & 2822 & 16 & 91,68 & $\mid 2822$ & 16 & $1122,28 \mid$ & & & $\infty$ \\
\hline xmodem & 2716 & 6 & 88,77 & 3269 & 6 & 256,61 & 3269 & 6 & 326,55 & 4806 & 6 & 802,53 \\
\hline
\end{tabular}

Fig. 4. Copy Constant Propagation

functional approach $(\mathcal{F})$. For each analysis method tree numbers are given: as a measurement of (theoretical) precision the number of available constants in the program is used. This is the number of constant variables at all control flow nodes, i.e.

$$
\sum_{n \in V} \mid\left\{\operatorname{var} \mid\left(\sqcup_{i=1, \ldots, \operatorname{Arity}(n)} \text { flow }(n)[i]\right)(\operatorname{var}) \neq \perp, \top\right\} \mid
$$

As a measurement of the usability of the calculated information the number of source code transformations is given that can be done with the information obtained by the constant propagation. This is the number of replacements of variables by their values. The last number is the runtime in seconds on a SUN SPARCsystem-600 (Ross-RT625 cpu, 128Mb memory), running SunOS 4.1.4.

To compare the different analysis approaches for each analysis three bar charts are used: Fig. 6, 7, 8 for the copy constant propagation and Fig. 9, 10, 11 


\begin{tabular}{|c|c|c|c|c|c|c|c|c|c|c|c|c|}
\hline Prog & Prec & $\begin{array}{c}\mathcal{C}_{0} \\
\text { Fold }\end{array}$ & Time & Prec & $\begin{array}{c}\mathcal{C}_{1} \\
\text { Fold }\end{array}$ & Time & Prec| & $\begin{array}{r}\mathcal{C}_{2} \\
\text { Fold }\end{array}$ & Time & Prec $\mid 1$ & $\begin{array}{c}\mathcal{F} \\
\mid \text { Fold }\end{array}$ & Time \\
\hline \begin{tabular}{|l|l}
$\operatorname{cdecl}$ \\
\end{tabular} & 885 & 10 & 15,93 & 903 & 10 & 65,08 & 911 & 10 & 135,02 & \begin{tabular}{l|}
911 \\
\end{tabular} & 10 & 101,05 \\
\hline twig & 995 & 6 & 8,31 & 1130 & 6 & 47,05 & 1156 & 6 & 230,13 & 1258 & 6 & 1813,02 \\
\hline dhry & 87 & 3 & 1,26 & 141 & 15 & 1,49 & 141 & 15 & 1,43 & 141 & 15 & 1,49 \\
\hline ed & 2503 & 50 & 6,99 & 2714 & 52 & 24,56 & 2763 & 52 & 30,63 & 2763 & 52 & 21,58 \\
\hline cloop & 10367 & 149 & 21,90 & 10711 & 149 & 260,06 & 10803 & 149 & 728,21 & 21952 & 151 & 2402,53 \\
\hline flex & 4329 & 22 & 16,82 & 4597 & 22 & 94,54 & 10890 & 24 & 397,14 & 10911 & 24 & 209,26 \\
\hline flops & 7080 & 188 & 0,74 & 7306 & 217 & 0,58 & & & & 7306 & 219 & 0,67 \\
\hline bison & 5591 & 10 & 24,03 & 5960 & 10 & 152,13 & 55973 & 10 & 1674,14 & & & $\infty$ \\
\hline linpack & 385 & 80 & 4,57 & 385 & 80 & 10,15 & 385 & 80 & 16,64 & 385 & 80 & 5,31 \\
\hline gzip & 4117 & 24 & 8,53 & 4916 & 24 & 83,00 & 5321 & 24 & 1604,51 & & & $\infty$ \\
\hline xmodem & 3870 & 12 & 94,23 & 4756 & 12 & 226,18 & 4756 & 12 & 323,80 & 7521 & 12 & 593,11 \\
\hline
\end{tabular}

Fig. 5. Full Constant Propagation

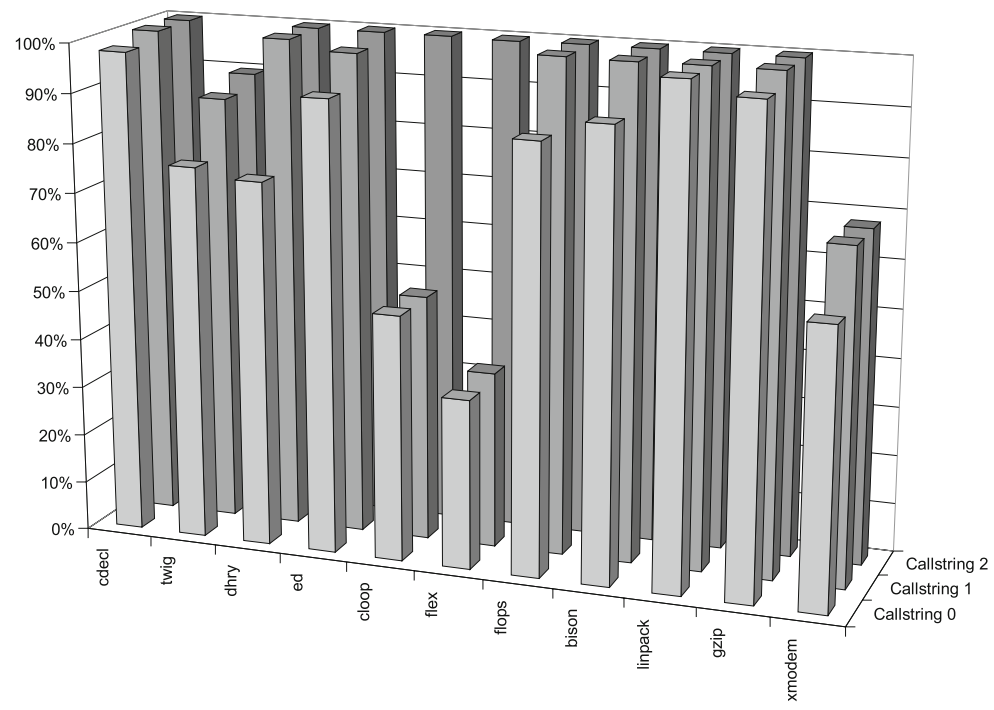

Fig. 6. Copy Constant Propagation: Percentage of available constants compared to the functional approach 


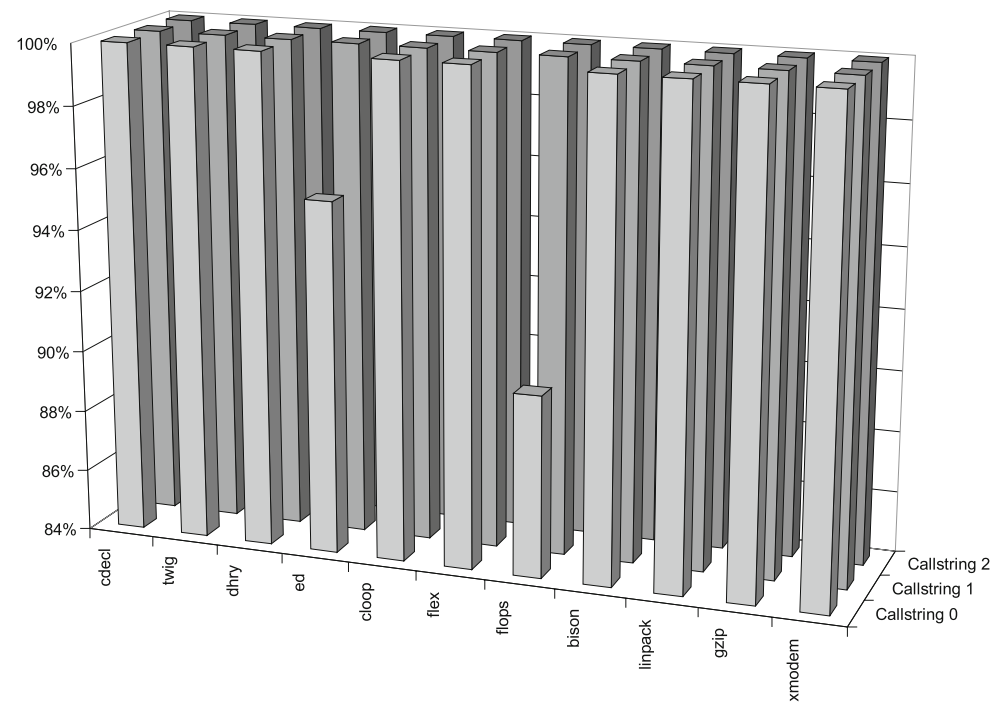

Fig. 7. Copy Constant Propagation: Percentage of constant foldings compared to the functional approach

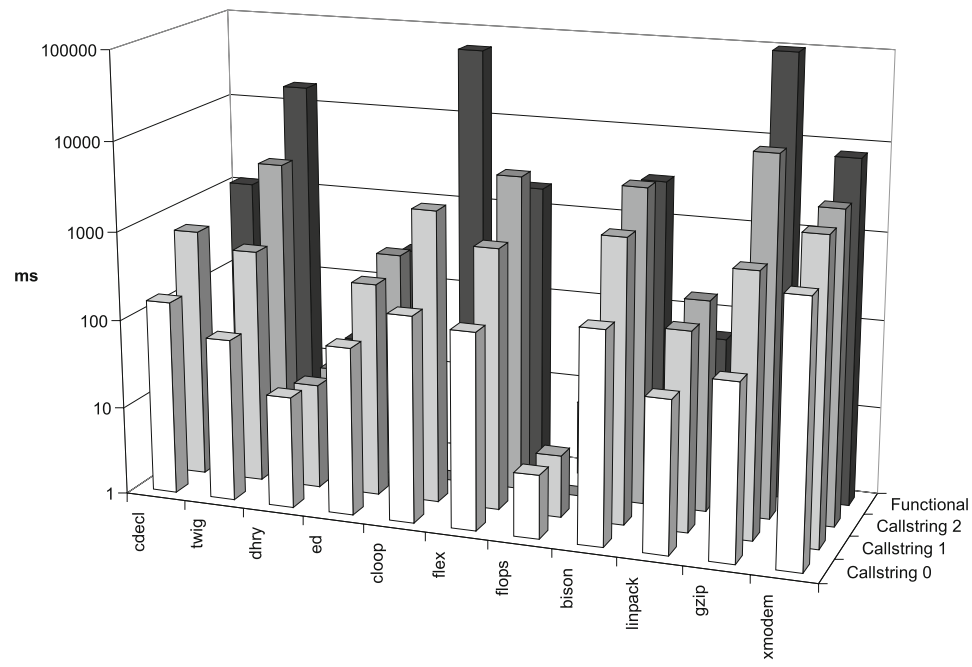

Fig. 8. Copy Constant Propagation: Runtimes 


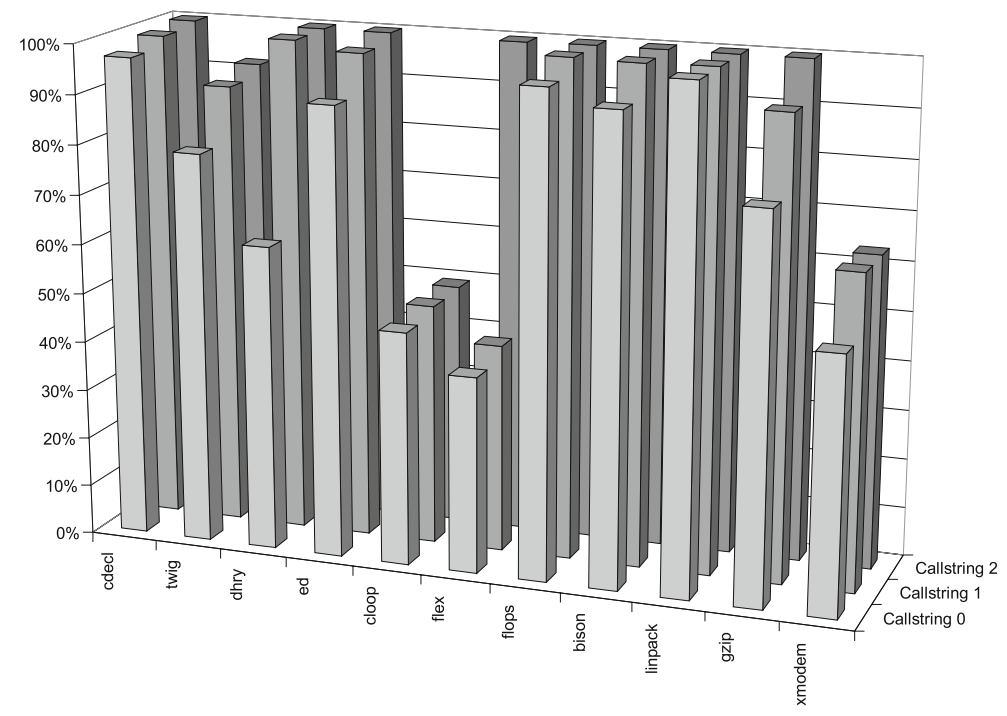

Fig. 9. Full Constant Propagation: Percentage of available constants compared to the functional approach

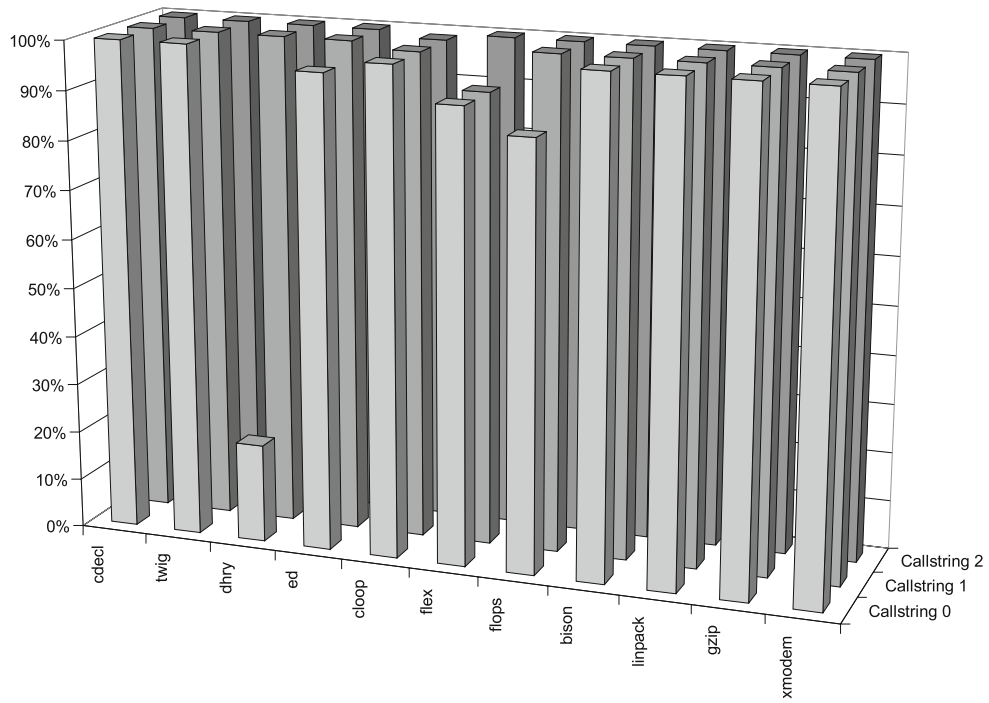

Fig. 10. Full Constant Propagation: Percentage of constant foldings compared to the functional approach 


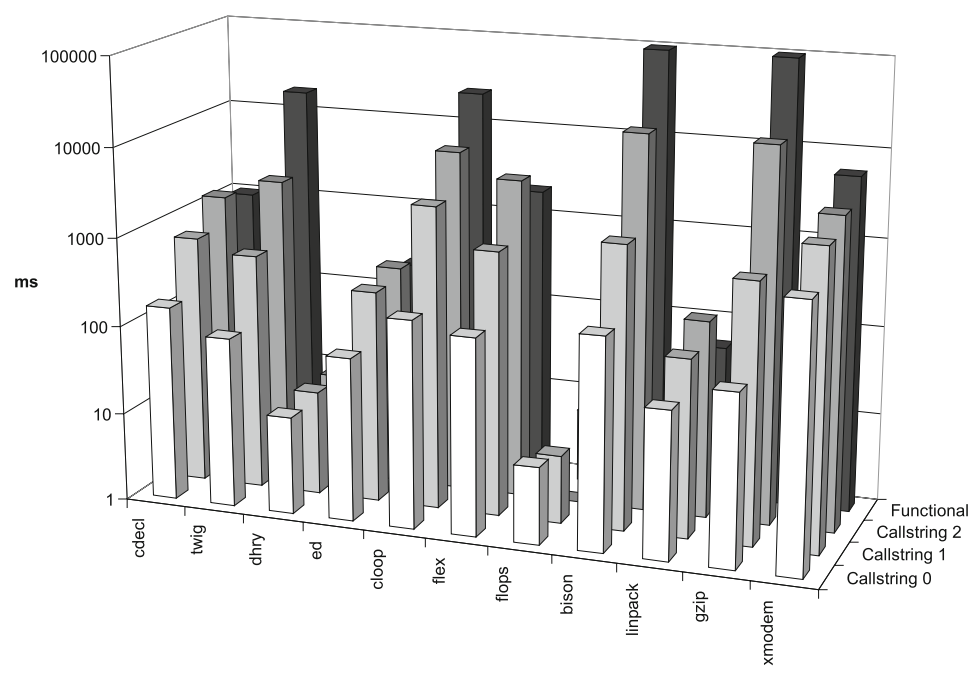

Fig. 11. Full Constant Propagation: Runtimes

for the full constant propagation. The first bar charts (Fig. 6, 9) show the relative number of available constants of the call string approaches compared to the functional approach, the second bar charts (Fig. 7, 10) show the same comparison for the number of foldings, and the third bar charts (Fig. 8, 11) show the runtime of the different approaches.

\subsection{Results}

As can be seen in (Fig. 4, 5) the functional analyzers find always the largest number of constants, if they terminate. As termination is not guaranteed for the full constant propagation and the functional approach, one would not use this in a production compiler. One can also see that runtimes of the functional analyzer are unsatisfying for programs like twig, cloop, bison, and gzip.

This can be understood by the discussion from Sect. 2.3, where it is stated, that the runtime of the functional analyzer depends on the locality properties of the analysis and the programs, as well as the programming language. And these are very bad for full ANSI C and the constant propagation.

So one can deduce from the theoretical discussion that the functional analyzer may have bad runtimes for some programs, and in fact this claim is supported by the practical measurements.

On the other hand one does not expect runtime explosions for the call string analyzer for $\mathrm{K}$ equals zero and one, since then the number of runs through a 
procedure is determined by one or the number of call sites to the procedure, which is small, at least for hand written programs.

The shortcoming of the call string approach is that it may deliver potentially worse analysis results than the functional approach. Also this claim can be verified by the measurements for the theoretical precision of the analyses (Fig. 6, 9). But the practical use that can be gained from this higher precision is very low. Only for cloop and flex in the full constant propagation the number of foldings is higher for the functional approach than for the call string 1 approach (Fig. 10).

One can also see from the experiments that the full constant propagation delivers more foldings than a copy constant propagation, and has only a minor impact on the run times (Fig. 4, 5).

\section{Conclusion and Further Work}

Although no new ideas are presented in this paper, it presents a fair experimental evaluation of the two techniques, call string- and functional approach for interprocedural data flow analysis applied to two data flow problems.

We have shown that a full constant propagation delivers better results than a simple one (Fig. 4 and 5). Furthermore one can see that the set of call strings which should be kept separately (1) is small for real programs, but leads to similar results as the functional approach.

So we can draw the conclusion that an expensive functional approach can be replaced by a cheaper call string approach, at least for constant propagation in languages similar to $\mathrm{C}$.

In the future we will test if these results also hold for other analyses.

\section{Acknowledgments}

I like to thank Martin Alt for contributing to early versions of this article, and Reinhard Wilhelm, Marc Langenbach and Christian Probst for careful proof reading.

\section{References}

Alt et al., 1996. Alt, M., Ferdinand, C., Martin, F., and Wilhelm, R. (1996). Cache Behavior Prediction by Abstract Interpretation. In Cousot, R. and Schmidt, D. A., editors, SAS'96, Static Analysis Symposium, volume 1145 of Lecture Notes in Computer Science, pages 51-66. Springer. Long version accepted for SAS'96 special issue of Science of Computer Programming.

Alt and Martin, 1995. Alt, M. and Martin, F. (1995). Generation of Efficient Interprocedural Analyzers with PAG. In Mycroft, A., editor, SAS'95, Static Analysis Symposium, volume 983 of Lecture Notes in Computer Science, pages 33-50. Springer.

Cousot and Cousot, 1992. Cousot, P. and Cousot, R. (1992). Abstract interpretation frameworks. Journal of Logic Computation, 2(4):511-547. 68 
Fecht, 1997. Fecht, C. (1997). Abstrakte interpretation logischer programme: Theorie, implementierung, generierung. Dissertation, Universität des Saarlandes, Fachbereich 14.67

Kam and Ullman, 1977. Kam, J. and Ullman, J. D. (1977). Monotone Data Flow Analysis Frameworks. Acta Informatica, 7:305-317. 64, 65

Knoop and Steffen, 1992. Knoop, J. and Steffen, B. (1992). The Interprocedural Coincidence Theorem. In Proceedings of the 4th International Conference on Compiler Construction, volume 641 of Lecture Notes in Computer Science, pages 125-140. Springer. 65

Martin, 1998. Martin, F. (1998). PAG - an efficient program analyzer generator. International Journal on Software Tools for Technology Transfer, Special Issue on Program Analysis. to appear. 64

Martin et al., 1998. Martin, F., Alt, M., Ferdinand, C., and Wilhelm, R. (1998). Analysis of Loops. In Koskimies, K., editor, Proceedings of the 7th International Conference on Compiler Construction, volume 1383 of Lecture Notes in Computer Science, pages 80-94. Springer. 66

Metzger and Stroud, 1993. Metzger, R. and Stroud, S. (1993). Interprocedural Constant Propagation: An Empirical Study. ACM Letters on Programming Languages and Systems, 2(1-4):213-232. 63

Nielson et al., 1998. Nielson, F., Nielson, H. R., and Hankin, C. L. (1998). Principles of Program Analysis - Flows and Effects. Preliminary version November 1998; complete version to appear 1999. 67

Reps et al., 1995. Reps, T., Horwitz, S., and Sagiv, M. (1995). Precise interprocedural dataflow analysis via graph reachability. In Conference Record of POPL '95: 22nd ACM SIGPLAN-SIGACT Symposium on Principles of Programming Languages, pages 49-61, San Francisco, California.

Sagiv et al., 1995. Sagiv, M., Reps, T., and Horwitz, S. (1995). Precise interprocedural dataflow analysis with application to constant propagation. In TAPSOFT'95, Arhus, Denmark, LNCS. Springer-Verlag. 63

Sharir and Pnueli, 1981. Sharir, M. and Pnueli, A. (1981). Two approaches to interprocedural data flow analysis. In Muchnick, S. S. and Jones, N. D., editors, Program Flow Analysis: Theory and Applications, chapter 7, pages 189-233. Prentice-Hall. 63

Thesing et al., 1998. Thesing, S., Martin, F., Lauer, O., and Alt, M. (1998). PAG User's Manual.

Wilhelm and Maurer, 1995. Wilhelm, R. and Maurer, D. (1995). Compiler Design. International Computer Science Series. Addison-Wesley. 